\title{
Research Square \\ Applications of Fuzzy Graph Theory Portrayed in Various Fields
}

\section{Abdul Muneera ( $\nabla$ scholar.abdulmuneera@gmail.com )}

Andhra Loyola Institute of Engineering and Technology

\section{T. Nageswara Rao}

Koneru Lakshmaiah Education Foundation

\section{R. V. N. Srinivasa Rao}

Wollega University

\section{J. Venkateswara Rao}

United States International University USIU Africa

\section{Research Article}

Keywords: Fuzzy Graph, Domination of fuzzy graph, Edge Domination of fuzzy graph

Posted Date: March 19th, 2021

DOl: https://doi.org/10.21203/rs.3.rs-298268/v1

License: (c) (i) This work is licensed under a Creative Commons Attribution 4.0 International License. Read Full License 


\title{
APPLICATIONS OF FUZZY GRAPH THEORY PORTRAYED IN VARIOUS FIELDS
}

\author{
Abdul. Muneera ${ }^{1}$, Dr. T. Nageswara Rao ${ }^{2}$, Dr. R. V. N. Srinivasa Rao ${ }^{3}$, \\ Dr. J. Venkateswara Rao ${ }^{4}$ \\ ${ }^{1}$ Research Scholar, KoneruLakshmaiah Education Foundation, Vaddeswaram, Guntur, \\ Andhra Pradesh, India. \\ ${ }^{2}$ Associate Professor, Deparatment of Mathematics, KoneruLakshmaiah Education \\ Foundation, Vaddeswaram, Guntur, Andhra Pradesh, India. \\ Email:tnraothota@kluniversity.in \\ ${ }^{3}$ Professor, Department of Mathematics, Wollega University Ethopia. \\ ${ }^{4}$ Professor, Department of Mathematics, School of Science \& Technology United States \\ International University USIU - Africa.
}

\begin{abstract}
The intend of the paper is to grant the centrality of fuzzy graph (f-graph) hypothetical ideas and the uses of dominations in fuzzy graphs to different genuine circumstances in the territories of science and designing. It is seen an eminent development because of various applications in PC and correspondence, biomedical, atomic material science and science, interpersonal organizations, natural sciences and in different various regions. Interpersonal organizations are the zones where countless individuals are associated. A wireless sensor Network (WSN) remote system which comprises of spatially circulated independent sensors to screen the physical or ecological conditions, for example, pressure, temperature, sound and so forth and to communicate their data through the remote system to a fundamental area. This paper gives an audit of the employments of Fuzzy Graph theory in different sorts of fields.
\end{abstract}

Keywords: Fuzzy Graph, Domination of fuzzy graph, Edge Domination of fuzzy graph.

\section{INTRODUCTION}

One of the unmistakable scientific innovations of the twentieth century is that of Fuzzy sets by Lotfi. A. Zadeh [1] in 1965. His point was to stretch out a mathematical theory to manage vulnerability and imprecision. To deal with the imprecise idea, the regular strategy for set theory and numbers are insufficient and should be reached out to some different ideas. A fuzzy set is characterized scientifically by allotting to every conceivable individual known to man of talk a worth, speaking to its evaluation of participation, which compares to the degree, to which that individual is comparative or good with the idea spoke to by the Fuzzy set. The upside of supplanting the traditional sets by Zadeh's fluffy sets is that it gives more exactness and accuracy in principle and more proficiency and framework similarity in applications. The variation among set and fuzzy set is that the set separation the general set into two subsets, to be specific individuals and non-individuals while fuzzy set appoints a succession of participation esteems to components of the all inclusive set extending from 0 to 1 . The principal meaning of Fuzzy graph by Kaufman (1973) depended on Zadeh's fuzzyrelations (1971). After that Rosenfeld (1975) [2] built up the theory of fuzzy graphs. Fuzzy graphs have numerous applications in diverse parts of Science and Engineering like broadcast communications, producing, Social Network, man-made reasoning, data hypothesis, neural systems and arranging and so forth.

Over the last 3 Decades, an eminent development has been found in graph theoryas of its expansive scope of utilizations in traditional combinatorial issues, arithmetical issues, and computational issues and so on. This is for the most part because of the expansion of various parameters that has been created from the essential meaning of domination. The idea of 
domination in graphs discovered its starting point in 1850s with the enthusiasm of a few chess players. Chess fans in Europe considered the issue of decide the base number of sovereigns that can be set on a chess board to that all the squares are either assaulted by a sovereign or involved by a sovereign. The control number is presented by Cockayne and Hedetniemi [3]. Among the various utilizations of the domination theory in Fuzzy graphs, the regularly talked about is a communication network. The issue is to choose a smallest arrangement of localities at which the transmitters are set with the goal that each other site in the system is joined by an immediate correspondence connect to the site, which as a transmitter. Such huge numbers of utilizations of Fuzzy Graph in Social Network like Face book, Twitter, WhatsApp, Research Gate, Instagram expanding step by step.

\section{PRELIMINARIES}

It is realized that graphs are models or affiliations. A graph is an advantageous method for speaking to data including connection between objects. The objects are represented by nodes and relations by arcs. Whenever there is ambiguity or vagueness in the description of items or in its connections or in both, it is common that we have to plan a fuzzy graph model.

Definition: 2.1 A fuzzy Graph or f-graph $\mathrm{G}(\xi, \eta)$ is set with 2 functions, $\xi: \mathrm{V} \rightarrow[0,1]$ and $\eta$ : $\mathrm{E} \rightarrow[0,1]$ to such an extent that $\eta(\mathrm{x} y) \leq \xi(\mathrm{x}) \wedge \xi(\mathrm{y})$ For all $\mathrm{x}, \mathrm{y} \in \mathrm{V}$.

Definition: 2.2 Let $\mathrm{G}(\xi, \eta)$ be a f-graph on V and V1 $\subseteq$ V. Characterize $\xi 1=\xi(\mathrm{x}) \forall \mathrm{x} \in \mathrm{V} 1 \&$ $\eta 1$ lying on the assortment E1 of 2component subsets of $V 1$ by $\eta 1(x y)=\eta(x y) \forall x, y \in V 1$. At that point $(\xi 1, \eta 1)$ is known as the fuzzy sub graph of $\mathrm{G}$ actuated by $\mathrm{V} 1 \&$ is indicated by $<\mathrm{V} 1>$.

Definition: 2.3 The order $m$ and size $n$ of a f-graph $G(\xi, \eta)$ are characterized to be $m=$ $\sum_{x \in V} \xi(\mathrm{x})$ and $\mathrm{n}=\sum_{x y \epsilon E} \eta(\mathrm{x} \mathrm{y})$.

Definition: 2.4 Let $\mathrm{G}(\xi, \eta)$ be a f-graph of $\mathrm{V}$ and $\mathrm{S} \subseteq \mathrm{V}$. At that point the fuzzy cardinality of $\mathrm{S}$ is characterized to be $\sum_{v \in S} \xi(\mathrm{V})$.

Definition: 2.5 Let us consider the fuzzy graph or a f-graph $\mathrm{G}(\xi, \eta)$ on $\mathrm{E}$ and $\mathrm{D} \subseteq \mathrm{E}$. At that point the fuzzy edge cardinality of $\mathrm{D}$ is characterized to be $\sum_{e \in D} \eta(\mathrm{e})$.

Definition: 2.6Consider a fuzzy graphG $(\xi, \eta)$. Characterize the degree of a node $v$ to be $d(v)$ $=\sum_{\eta \neq V} \eta(\mathrm{u}, \mathrm{v})$. The minimum (least value) degree of f-graph $\mathrm{G}$ is $\delta(\mathrm{G})=\wedge\{\mathrm{d}(\mathrm{v}) / \mathrm{v} \in \mathrm{V}\}$ and the maximum (greatest value) degree of f-graph $\mathrm{G}$ is $\Delta(\mathrm{G})=\mathrm{V}\{\mathrm{d}(\mathrm{v}) / \mathrm{v} \in \mathrm{V}\}$.

Definition: 2.7 An arc e $=(\mathrm{v} w)$ of a f-graph is called an effective edge if $\eta(\mathrm{v}, \mathrm{w})=\xi(\mathrm{v}) \wedge \xi$ $(\mathrm{w}) . \mathrm{N}(\mathrm{v})=\{\mathrm{w} \in \mathrm{V} /(\mathrm{v} \mathrm{w})=\xi(\mathrm{v}) \wedge \xi(\mathrm{w})$ is known as the area of $\mathrm{v}$ and $\mathrm{N}[\mathrm{v}]=\mathrm{N}(\mathrm{v}) \cup\{\mathrm{v}\}$ is the closed neighborhood of $\mathrm{v}$.

Definition: 2.8Consider a fuzzy graph $\mathrm{G}(\xi, \eta)$. $\mathrm{S} \subseteq \mathrm{V}$ is assumed to be dominating set of $\mathrm{G}$ if intended for each $v \in V-S \exists$ a component $u \in S$ with the end goal that $\eta(u, v)=\xi(u) \wedge \xi$ (v). An overwhelming set $\mathrm{S}$ of $\mathrm{G}$ is known as the insignificant commanding arrangement of $\mathrm{G}$ if each node $\mathrm{v} \in \mathrm{S}, \mathrm{S}-\{\mathrm{v}\}$ is definitely not a dominating set. The minimum scalar cardinality of $\mathrm{S}$ is known as the domination number and it is indicated by $\gamma(\mathrm{G})$.

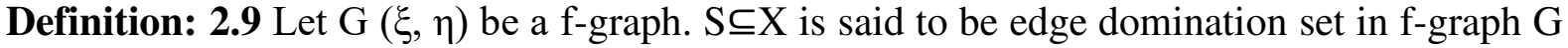
if for each or arc in $\mathrm{X}-\mathrm{S}$ is neighboring to as a minimum any one effective edge in $\mathrm{S}$. The Lowest (minimum) Fuzzy cardinality of an edge domination set $\mathrm{G}$ is known as the edge domination number of $\mathrm{G}$ and is indicated by $\gamma^{\prime}(\mathrm{G})$.

\section{APPLICATIONS IN VARIOUS FIELDS}

\subsection{Utility of Fuzzy Graph in Medical Field}

Utilizations of Artificial Intelligence Techniques occurred in numerous zones including medication, for example, determination, treatment of sickness, tolerant interest, and expectation of illness chance and so on. Fuzzy logic approach, as opposed to a certain or 
parallel rationale, utilizes a rationale and decision mechanism which doesn't have certain limits like human rationale. At the point when an individual is given a clinical assessment, a wide assortment of parameters, called side effects in clinical language, can be found out and estimated. Because of the intricacy of the human body, it is beyond the realm of imagination to expect to give a sensible utmost for the quantity of built up criteria. Fuzzy set theory rationale is a scientific control that we use evey day and causes us to arrive at the structure in which we decipher our own practices. Fuzzy set theory in which esteems among genuine and bogus that is halfway valid and in part bogus are resolved. Fuzzy set theory express the vulnerabilities of life, for example, warm and cool which are in the middle of hot and cold scientifically. At the point when a specialist begins treatment of a patient he utilizes his own understanding, information from books, and mental capacity. The specialist takes note of the patient's signs and side effects, joins these with the patient's clinical history, physical assessment and laboratory findings, and afterward analyzes the diseases. Along these lines, the objective of the fuzzy intelligent system is to impersonate conduct of a specialist and give him consultation.

Graphs have been generalized to hyper graphs, which have been generally and profoundly contemplated in Berge's inquires about $(1973,1984,1989)$, and frequently have end up being effective apparatus to speak to and reproduction ideas and structures in different territories of Computer Science. In a Graph, an edge relates just a couple of focuses, yet the edges of the hyper graph known as hyper edges can relate gatherings of multiple focuses. In a correspondence arrange spoke to as a simple graph we just know whether two individuals have imparted or not, however we can't know whether at least three individuals connected together in the system have traded a similar message. Vertices speak to as individuals in hyper graph and hyper edges speak to the gathering of individuals who traded a similar message in Communication arrange. There are numerous issues in various fields that can be tackled utilizing soundness of fuzzy hyper graphs. One of these issues is looked in the clinical field when we use X-ray CT registered tomography to analyze some diseases, for example, Cancer. CT scan is considered of an enormous number of people, hairline-flimsy X-beam bars that lie in the plane of the cross segment. After they go through the cross area, a X-beam locator gauges the powers of the X-beam pillars and these estimations are as for a PC where they are handled. The field of view wherein the cross area is arranged has been isolated into many square pixels numbered 1 through $\mathrm{N}$.

\subsection{Telecommunication Framework Dependent on Fuzzy Graphs}

Communication is the fundamental parts of human way of life. Presently in our everyday life Telecommunication is one of required and unavoidable framework. Media transmission or Telecommunication is the trading of data over any separation by a media transmission way. Fuzzy idea is utilized to compute the beating likelihood of clients. Stir is a term utilized by organizations to signify the loss of purchasers. To distinguish agitating people fuzzy telecommunication network (FTN) is presented which is structured by fuzzy graph theory.

Let $\mathrm{X}=\{\mathrm{v} 1, \mathrm{v} 2, ., \mathrm{vn}\}$, where $\mathrm{n}$ is an enormous whole number, be the arrangement of every enrolled customer in the media transmission organize Fuzzy telecommunication network and $\mathrm{Y}=\{\mathrm{vn}+1, \mathrm{vn}+2, \ldots . \mathrm{vk}\}$ be the outside customers associated with the individuals fromFuzzy telecommunication network. Let $\mathrm{V}=\mathrm{XU} \mathrm{Y}$. The enrollment estimations of the customers are given by $\mathrm{k}: \mathrm{V} \rightarrow[0,1]$ and the participation estimations of the connections between the customers are given by $\mu: \mathrm{V} \times \mathrm{V} \rightarrow[0,1]$. At that point, the telecommunication system is represented by a coordinated fuzzygraph $\mathrm{G}^{*}=(\mathrm{V}, \mathrm{k}, \mu)$. The basic f-graph of $\mathrm{G}^{*}$ is signified by $\mathrm{G}^{*}=(\mathrm{V}, \xi, \eta)$, where $\mu: \mathrm{V} \times \mathrm{V} \rightarrow[0,1]$ to such an extent that $\mu \overline{(u, v)}=(\mu(\mathrm{u}, \mathrm{v})+\mu(\mathrm{v}, \mathrm{u})) / 2$ for all $\mathrm{u}, \mathrm{v} \in \mathrm{V}$. 
Two individuals are extremely close in media transmission when they talk additional time over phones. So quality between two companions relies upon how a lot of time they call to one another by telephones for each unit intervalof time. We mean $\overline{\left(C_{i}, C_{j}\right)}$, if $\mathrm{C}_{\mathrm{i}}$ calls $\mathrm{C}_{\mathrm{j}}$ by telephones where $\mathrm{Ci}, \mathrm{Cj} \in \mathrm{V}$. Let $\mu: \mathrm{V} \times \mathrm{V} \rightarrow[0,1]$ be a mapping with the end goal that

$$
\mu\left(\mathrm{C}_{\mathrm{i}}, \mathrm{C}_{\mathrm{j}}\right)=\left\{\begin{array}{c}
\frac{t}{T}\left(\sigma\left(C_{i}\right) \wedge \sigma\left(C_{j}\right)\right), \text { if } t \in[0, T] \\
\sigma\left(C_{i}\right) \wedge \sigma\left(C_{j}\right), \text { if } t>T .
\end{array}\right.
$$

Where $t$ is the term of call per unit interval of time and $T$, fulfilled time of calling is fixed positive genuine number for a system. We signify $\mu(\mathrm{Ci}, \mathrm{Cj})$ as the membership value of the link $\left.\overline{\left(C_{i} C_{j}\right.}\right)$. Henceforth $\mathrm{G}^{*}=(\mathrm{V}, \xi, \eta)$ speak to Fuzzy Telecommunication Network framework.

\subsection{Exploit of Fuzzy Graph in Traffic Light Control}

The control strategy of the traffic light relies generally upon the quantity of vehicles in the crossing point line. On the off chance that the traffic stream in the crossing point line is high, at that point there is a chance of mishap. At the point when the quantity of vehicles in the crossing point line is low then there might be less chance of mishap. The idea of mishap and number of vehicles in each line could be fuzzy. This shouldn't be numerical, is related to the ideal security level for the traffic. Here we describe each traffic stream with a fuzzy edge whose enrollment esteem relies upon the quantity of vehicles in that way. Two fuzzy nodes are neighboring on the off chance that the relating traffic streams cross one another; at that point there is a chance of mishap. Plausibility of mishap worth will rely upon node enrollment esteem. The most extreme security level is achieved when all paths are viewed as in crossing point with one another and the quantity of vehicles in each line is likewise high. So Graph will be a complete graph. Right now, chromatic number is the quantity of paths and the control approach of the lights guarantee that just a single development is permitted in any space of the cycle. Then again, the base security level is achieved when the crossing point edge set is unfilled, right now, chromatic number is 1 and all developments are permitted at any moment.

We speak to each traffic stream with a node and their membership value. Two nodes are adjacent if the relating traffic streams cross one another. For example, course $\mathrm{C}$ and $\mathrm{H}$ converges, so nodes $\mathrm{C}$ and $\mathrm{H}$ are contiguous (intersect). In the event that two nodes are neighboring, at that point there is a chance of mishap. The chance of mishap relies upon the adjacent nodes enrollment esteem. In the event that participation estimation of the adjacent two nodes is high $(\mathrm{H})$ at that point there is greater chance of mishap. So we consider participation estimation of that bend is high $(\mathrm{H})$. On the off chance that enrollment estimation of one adjacent node is high $(\mathrm{H})$ and another is low (L) at that point we consider the participation estimation of that curve is medium (M). On the off chance that enrollment estimation of the neighboring two hubs is medium (M) at that point we consider participation estimation of that bend is medium (M).

\subsection{Utilize of Fuzzy Graph in Neural Networks}

Neural systems are disentangled models of the organic sensory system and in this way have drawn their motivation from the sort of registering performed by a human mind. Neural systems exhibit trademark, for example, mapping abilities or example affiliation, speculation, vigor, adaptation to internal failure, and resemble and rapid data handling. Fuzzy neural systems and neural fuzzy frameworks are ground-breaking procedures for different computational and control applications. The region is still under an extraordinary deluge from both hypothetical and applied research. There is no orderly or brought together methodology for fusing the ideas of fuzziness and neural handling. Fuzzy sets can be utilized to delineate 
different parts of Neural Computing. That is, fuzziness might be presented at the info yield signals, synaptic loads, and collection activity and actuation capacity of individual neurons to make it fluffy neuron. Diverse collection tasks and actuation capacities bring about fuzzy neurons with various properties. Accordingly there are numerous prospects for fuzzification of a counterfeit neuron.

Applying fuzzy techniques into the activities of neural systems establishes a significant push of neuron-fuzzy computing. A fuzzy neuron has a similar fundamental structure as the counterfeit neuron with the exception of that its segments and parameters are depicted through the arithmetic of fuzzy logic. There are numerous prospects for fuzzification of a fake neuron so we may discover an assortment of fuzzy neurons in the literature. A fuzzyy neuron comprises of outer data sources, neurotransmitters/synaptic loads, dendrites, soma, and an axon through which the neural yield is transmitted to different neurons. The outer sources of info v1, v2,...,vn enter the jth neuron and gets adjusted by the synaptic loads wj1, wj2, ... ..wjn. Each synaptic yield frames a contribution to the handling component (soma), called the dendritic info. The outer info $\mathrm{v} 1, \mathrm{v} 2, \ldots$., vn are of fuzzy signs limited by participation esteems over the interval $[0,1]$. The synaptic loads wji are likewise characterized over the interval $[0,1]$.

\section{Uses of Domination Concept}

The use of domination in graph lies in different fields in taking care of genuine issues. It incorporates interpersonal organization theory, land reviewing, radio broadcasts, PC correspondence systems, school transport directing, interconnection systems and so on.

\subsection{Social Network Theory}

The online interpersonal organization has been grown essentially in the ongoing years as a mode of correspondence, sharing the data and spreading the impact. The issue of using the online informal community for taking care of the social issues in the physical world, for example, drinking, smoking, and medication issues are totally investigated well.

The dominating set assumes a fundamental job in examining the impact on a genuine online informal organization informational index through reproduction. The domination set idea can be applied to the interpersonal organization graph to decide the measure of constructive impact that is controlled by a person just as its effect on their related neighbor. A f-graph $G=(\sigma, \mu, c)$ is utilized to mean the online interpersonal organization, since kinship in an online informal community are typically bidirectional. Online informal organization can be spoken to as a graph of correlation with people speaking to the vertices of a graph, the social collaborations as arcs \& $\mathrm{C}$ is the partition vector that spares the partition of every vertex. The partition part of a vertex chooses whether the social issues of an individual has $+v e$ or - ve effect on its neighbors.

\subsection{Dominating Set in Wireless Sensor Network}

A WSN (Wireless Sensor Network) is a sort of remote system which comprises of spatially disseminated self-ruling sensors to screen the physical or ecological circumstances, for example, Pressure, sound, temperature and so on and to communicate their data through the remote system to primary area. The principle action in the WSN structures the convenient steering of data between the nodes. Various directing methods are accessible for remote sensor systems. Among them, various leveled steering and group based directing with virtual backbone framework is fundamentally utilized to execute vitality effective directing is WSN. Along these lines, the CDS comes into depiction to fill in as a virtual spine for WSNs to diminish the steering expenses. The CDS joined various leveled strategies rearranges the directing by limiting the fundamental steering assignment to the dominating nodes as it were. 


\section{GPS or Google Maps}

GPS or Google Maps are to discover the briefest course starting with one goal then onto the next. The objectives are vertices and their associations are edges comprising separation. The ideal course is dictated by programming. Schools/universities are additionally utilizing this capability to get together understudies from their stop to class. To every one stop is a vertex and the course is an edge. A Hamiltonian path exhibits the productivity of remembering each vertex for the course.

\section{CONCLUSION}

This paper shows the significance of fuzzy graph hypothetical thoughts in different regions of the genuine field for the scientist. An outline is introduced especially to extend the idea of fuzzy Graph Theory. This paper is valuable for students and scientists to get an outline of fuzzy graph applications in different genuine fields like Compute Science, Biology and Geography. An informal community model has been presented with the assistance of fuzzy graph theory. Media transmission or Telecommunication network arranges is likewise spoken to by fuzzy diagrams. Stirring proportion of clients shows the clients stability in the system. In the event that an individual in unit by confirmation class gets full participation esteem, the individual can't be phony for the most part. In any case, on the off chance that an individual in unit by acknowledgment classification gets full participation esteem, at that point quite possibly the profile is phony.

\section{Declaration Statement}

None.

\section{REFERENCES}

[1] Fuzzy sets by Lotfi. A. Zadeh. (1965). Information and Control, 8, 338 - 353.

[2] Rosenfeld, A., Fuzzy graphs, ln: Zadeh, L. A., Fu, K. S., \& Shimura Eds, M. (1975). Fuzzy sets and their applications. Academic Press, New York, 77 - 95.

[3] Cockayne, E. J., Dawes, R.M., \& Hedetniemi, S. T. (1980). Total Domination in Graphs. Networks, 10, 211-219,

[4] Sasireka, A., Nandhu Kishore, A. H. (2014). Applications of Dominating Set of Graph in Computer Networks. International Journal of Engineering Sciences \& Research Technology, 3(1), 170 -173.

[5] Mordeson, J. N., Nair, P. S. (2000). Fuzzy Graphs and Hypergraphs. PhysicaVerlag Heidelberg, 46, 248.

[6] Sovan Samanta \& Madhumangal Pal. Telecommunication System Based on Fuzzy Graphs. Journal of Telecommunications System \& Management, 3(1).

[7] Ramprasad C., Varma P. L. N., Satyanarayana S., Srinivasarao N. (2017). Vertex Degrees and Isomorphic Properties in Complement of an m-Polar Fuzzy Graph. Advances in Fuzzy Systems, wos.

[8] Rajeswari, M., Amudhavel, J., Dhavachelvan, P. (2017). Vortex Search Algorithm For Solving Set Covering Problem In Wireless Sensor Network. Advances And Applications In Mathematical Sciences, 17(1), 95-111.wos

[9] Venkateswara Rao, V., Varma, P. L. N., Reddy Babu, D. (2018). Eccentric connectivity index and connective eccentric index w.r.t. detour D-distance. Journal of Advanced Research in Dynamical and Control Systems, 10(2), 466-472 wos

[10] Adilakshmi, G., Kishore, G. N. V., Sridhar, W. (2018). Some new CARISTI type results in metric spaces with an application to graph theory. International Journal of Engineering and Technology(UAE), 7(4.10 Special Issue 10), 303-305. 
[11] Leelavathi, R., Suresh Kumar, G., Murty, M. S. N. (2018). Nabla integral for fuzzy functions on time scales. International Journal of Applied Mathematics , 31(5), 669-680.

[12] Prasad, D. R., Kishore, G. N. V., Iayk, H., Rao, B. S., Lakshmi, G. A. (2019). C*algebra valued fuzzy soft metric spaces and results for hybrid pair of mappings. Axioms, $8(3), 2-18$.

[13] Muneera, A., Nageswara Rao, T., Vendateswara Rao, J., Srinivasa Rao, R. V. N. (2020). Domination in regular and irregular bipolar fuzzy graphs. Journal of Critical Reviews, 7 (11), 793-796.

[14] Amiripalli, Shanmuk Srinivas, Bobba, Veeramallu. (2019). Impact of trimet graph optimization topology on scalable networks. Journal Of Intelligent \& Fuzzy Systems, 36(3), 2431-2442. wos

[15] Atik, Fouzul, Bapat, Kannan, R. B., Rajesh, M. (2019). Resistance matrices of graphs with matrix weights. Linear Algebra And Its Applications, 571, 41-57.

[16] Vasavi, C. H., Kumar, G. S., Rao, T. S., Rao, B.V.A. (2017). Application of fuzzy differential equations for cooling problems. International Journal of Mechanical Engineering and Technology, 8(12), 712-721.

[17] Leelavathi, R., Suresh Kumar, G., Murty, M. S. N., Srinivasa Rao, R. V. N. (2019). Existence-uniqueness of solutions for fuzzy nabla initial value problems on time scales. Advances in Difference Equations, (1), wos.

[18] Muneera, A., Nageswara Rao, T. (2020). Split and non-split domination on anti-fuzzy graph. Journal of Advanced Research in Dynamical and Control systems, 12, 86-92.

[19] Leelavathi, R., Suresh Kumar, G. (2019). Characterization theorem for fuzzy functions on time scales under generalized nabla hukuhara difference. International Journal of Innovative Technology and Exploring Engineering, 8(8), 1704-1706.

[20] Vundavilli, P. R., Parappagoudar, M B., Kodali, S. P., Benguluri, S. (2012). Fuzzy logicbased expert system for prediction of depth of cut in abrasive water jet machining process. Knowledge-Based Systems, DOI:10.1016/j.knosys.2011.10.002. 